\title{
AN $N$-DIMENSIONAL VERSION OF THE BEURLING-AHLFORS EXTENSION
}

\author{
Leonid V. Kovalev and Jani Onninen \\ Syracuse University, Department of Mathematics \\ Syracuse, NY 13244, U.S.A.; lvkovale@syr.edu \\ Syracuse University, Department of Mathematics \\ Syracuse, NY 13244, U.S.A.; jkonnine@syr.edu
}

\begin{abstract}
We extend delta-monotone quasiconformal mappings from dimension $n$ to $n+1$ while preserving both monotonicity and quasiconformality. This gives an analytic proof of the extendability of quasiconformal mappings that can be factored into bi-Lipschitz and delta-monotone mappings. In the case $n=1$ our approach yields a refinement of the Beurling-Ahlfors extension.
\end{abstract}

\section{Introduction}

Extension problem. Given a mapping $f: \mathbf{R}^{n} \rightarrow \mathbf{R}^{n}$ of class $\mathscr{A}$, find $F: \mathbf{R}^{n+1} \rightarrow$ $\mathbf{R}^{n+1}$ of class $\mathscr{A}$ such that the restriction of $F$ to $\mathbf{R}^{n}$ agrees with $f$.

Let us introduce coordinate notation $x=\left(x^{1}, \ldots, x^{n}\right)$ and $f=\left(f^{1}, \ldots, f^{n}\right)$. By setting $F^{i}=f^{i}$ for $i=1, \ldots, n$ and $F^{n+1}=x^{n+1}$ one immediately obtains a solution to the extension problem for many classes $\mathscr{A}$ such as continuous $\left(\mathscr{A}=C^{0}\right)$, smooth $\left(\mathscr{A}=C^{k}\right)$, homeomorphic, diffeomorphic, and (bi-)Lipschitz mappings.

When $\mathscr{A}=\mathscr{Q} \mathscr{C}$, the class of quasiconformal mappings, the extension problem is much more difficult. It was solved

- for $n=1$ by Beurling and Ahlfors [4] in 1956,

- for $n=2$ by Ahlfors [1] in 1964,

- for $n \leq 3$ by Carleson [8] in 1974, and

- for all $n \geq 1$ by Tukia and Väisälä [16] in 1982 .

The Tukia-Väisälä extension uses, among other things, Sullivan's theory [15] of deformations of Lipschitz embeddings. Except for the low dimensional case $n \leq 2$, no analytic solution of the extension problem is available. Our goal is to give an explicit extension for the subgroup of $\mathscr{Q} \mathscr{C}$ generated by bi-Lipschitz and delta-monotone mappings. Quasiconformal mappings can be defined as orientation-preserving quasisymmetric mappings [11, 17].

Definition 1.1. A homeomorphism $f: \mathbf{R}^{n} \rightarrow \mathbf{R}^{n}$ is quasisymmetric if there is a homeomorphism $\eta:[0, \infty) \rightarrow[0, \infty)$ such that

$$
\frac{|f(x)-f(z)|}{|f(y)-f(z)|} \leq \eta\left(\frac{|x-z|}{|y-z|}\right)
$$

doi:10.5186/aasfm.2011.3620

2010 Mathematics Subject Classification: Primary 30C65; Secondary 47H05, 47 B34.

Key words: Quasiconformal mapping, extension, monotone mapping.

Kovalev was supported by the NSF grants DMS-0913474 and 0968756.

Onninen was supported by the NSF grants DMS-0701059 and 1001620. 
for $x, y, z \in \mathbf{R}^{n}, z \neq y$.

One can say that quasisymmetry is a three-point condition. But there are two subclasses of $\mathscr{Q} \mathscr{C}$ that are defined by two-point conditions, namely bi-Lipschitz class $\mathscr{B} \mathscr{L}$ and the class of nonconstant delta-monotone mappings [2, Chapter 3]. Recall that a mapping $f: \mathbf{R}^{n} \rightarrow \mathbf{R}^{n}$ is monotone if

$$
\langle f(x)-f(y), x-y\rangle \geq 0 \text { for all } x, y \in \mathbf{R}^{n} .
$$

We called $f$ delta-monotone if there exists $\delta>0$ such that

$$
\langle f(x)-f(y), x-y\rangle \geq \delta|f(x)-f(y)||x-y| \quad \text { for all } x, y \in \mathbf{R}^{n} .
$$

The class of nonconstant delta-monotone mappings is denoted by $\mathscr{D} \mathscr{M}$. When we want to specify the value of $\delta$ we write that $f$ is $\delta$-monotone.

In contrast to the bi-Lipschitz case, the extension problem for the class $\mathscr{D} \mathscr{M}$ cannot be solved by means of the trivial extension. For example, the mapping $f(x)=$ $|x|^{p} x, p>-1$, belongs to $\mathscr{D} \mathscr{M}$ but its trivial extension does not (unless $p=0$ ).

Main result. Let $n \geq 2$. For any mapping $f: \mathbf{R}^{n} \rightarrow \mathbf{R}^{n}$ of class $\mathscr{D} \mathscr{M}$ there exists $F: \mathbf{R}^{n+1} \rightarrow \mathbf{R}^{n+1}$ of class $\mathscr{D} \mathscr{M}$ such that the restriction of $F$ to $\mathbf{R}^{n}$ agrees with $f$.

Let $\mathscr{Q}{ }_{d} \subset \mathscr{Q} \mathscr{C}$ be the group generated by $\mathscr{B} \mathscr{L}$ and $\mathscr{D} \mathscr{M}$ under composition. In other words, $f$ belongs to $\mathscr{Q} \mathscr{C}_{d}$ if it can be factored into bi-Lipschitz and deltamonotone mappings. This should be compared with the notion of polar factorization of mappings introduced by Brenier [6]. By combining our main result with the trivial extension of bi-Lipschitz mappings, we obtain a solution to the extension problem for $\mathscr{Q} \mathscr{C}_{d}$.

Corollary 1.2. Let $n \geq 2$. For any mapping $f: \mathbf{R}^{n} \rightarrow \mathbf{R}^{n}$ of class $\mathscr{Q} \mathscr{C}_{d}$ there exists $F: \mathbf{R}^{n+1} \rightarrow \mathbf{R}^{n+1}$ of class $\mathscr{Q} \mathscr{C}_{d}$ such that the restriction of $F$ to $\mathbf{R}^{n}$ agrees with $f$.

Let us now describe the extension process, which can be viewed as an $n$-dimensional version of the Beurling-Ahlfors extension [4]. Suppose $f \in \mathscr{D} \mathscr{M}$. Let $\mathbf{R}_{+}^{n+1}=$ $\mathbf{R}^{n} \times[0, \infty)$ and

$$
\phi(x)=(2 \pi)^{-\frac{n}{2}} e^{-|x|^{2} / 2}, \quad x \in \mathbf{R}^{n} .
$$

We define $F: \mathbf{R}_{+}^{n+1} \rightarrow \mathbf{R}_{+}^{n+1}$ by

$$
\begin{aligned}
F^{i}(x, t) & =\int_{\mathbf{R}^{n}} f^{i}(x+t y) \phi(y) d y, \quad i=1, \ldots, n, \\
F^{n+1}(x, t) & =\int_{\mathbf{R}^{n}}\langle f(x+t y), y\rangle \phi(y) d y
\end{aligned}
$$

where $x \in \mathbf{R}^{n}, t \geq 0$ (see $\S 4$ for the convergence of these integrals). Observe that $F(x, 0)=(f(x), 0)$. Furthermore, $F^{n+1}(x, t) \geq 0$ because

$$
\int_{\mathbf{R}^{n}}\langle f(x+t y), y\rangle \phi(y) d y=\int_{\mathbf{R}^{n}}\langle f(x+t y)-f(x), y\rangle \phi(y) d y \geq 0
$$

due to the monotonicity of $f$. Finally, we extend $F$ to $\mathbf{R}^{n+1}$ by reflection

$$
F^{i}(x, t)=F^{i}(x,-t), \quad i=1, \ldots, n, \quad \text { and } \quad F^{n+1}(x, t)=-F^{n+1}(x,-t) .
$$


Theorem 1.3. Let $n \geq 2$. If $f: \mathbf{R}^{n} \rightarrow \mathbf{R}^{n}$ is $\delta$-monotone, then $F: \mathbf{R}^{n+1} \rightarrow \mathbf{R}^{n+1}$ is $\delta_{1}$-monotone where $\delta_{1}$ depends only on $\delta$ and $n$. In addition, $F: \mathbf{H}^{n+1} \rightarrow \mathbf{H}^{n+1}$ is bi-Lipschitz in the hyperbolic metric.

Here $\mathbf{H}^{n+1}=\mathbf{R}^{n} \times(0, \infty)$ and the hyperbolic metric on $\mathbf{H}^{n+1}$ is $|d x| / x^{n+1}$. Theorem 1.3 can be also formulated for $n=1$, in which case it becomes a refinement of the Beurling-Ahlfors extension theorem.

Proposition 1.4. If $f: \mathbf{R} \rightarrow \mathbf{R}$ is increasing and quasisymmetric, then $F: \mathbf{R}^{2} \rightarrow$ $\mathbf{R}^{2}$ is $\delta_{1}$-monotone where $\delta_{1}$ depends only on $\eta$ in Definition 1.1. Furthermore, $F: \mathbf{H}^{2} \rightarrow \mathbf{H}^{2}$ is bi-Lipschitz in the hyperbolic metric.

Fefferman, Kenig and Pipher [9, Lemma 4.4] proved that $F$ in Proposition 1.4 is quasiconformal. Proposition 1.4 was originally proved in [12] using their result. In this paper we give a direct proof.

Theorem 1.3 has an application to mappings with a convex potential [7], i.e., those of the form $f=\nabla u$ with $u$ convex. The basic properties and examples of quasiconformal mappings with a convex potential are given in [13].

Corollary 1.5. Suppose that $f: \mathbf{R}^{n} \rightarrow \mathbf{R}^{n}, n \geq 2$, is a $K$-quasiconformal mapping with a convex potential. Then $f$ can be extended to a $K_{1}$-quasiconformal mapping $F: \mathbf{R}^{n+1} \rightarrow \mathbf{R}^{n+1}$ with a convex potential, where $K_{1}$ depends only on $K$ and $n$.

\section{Preliminaries}

Let $e_{1}, \ldots, e_{n+1}$ be the standard basis of $\mathbf{R}^{n+1}$. All vectors are treated as column vectors. The transpose of a vector $v$ is denoted by $v^{T}$. We use the operator norm $\|\cdot\|$ for matrices. A Borel measure $\mu$ on $\mathbf{R}^{n}$ is doubling if there exists $\mathscr{D}_{\mu}$, called the doubling constant of $\mu$, such that

$$
\mu(2 B) \leq \mathscr{D}_{\mu} \mu(B)
$$

for all balls $B=B(x, r)$. Here $2 B=B(x, 2 r)$.

The geometric definition of class $\mathscr{Q} \mathscr{C}$ given in the introduction is equivalent to the following analytic definition $[11,17]$.

Definition 2.1. A homeomorphism $f: \mathbf{R}^{n} \rightarrow \mathbf{R}^{n}(n \geq 2)$ is quasiconformal if $f \in W_{\text {loc }}^{1, n}\left(\mathbf{R}^{n}, \mathbf{R}^{n}\right)$ and there exists a constant $K$ such that the differential matrix $D f(x)$ satisfies the distortion inequality

$$
\|D f(x)\|^{n} \leq K \operatorname{det} D f(x) \quad \text { a.e. in } \mathbf{R}^{n} .
$$

Delta-monotone mappings also have an analytic definition.

Lemma 2.2. Let $\Omega$ be a convex domain in $\mathbf{R}^{n}, n \geq 2$. Suppose $f \in W_{\text {loc }}^{1,1}\left(\Omega, \mathbf{R}^{n}\right)$ is continuous. The following are equivalent:

(i) $f$ is $\delta$-monotone in $\Omega$ for some $\delta>0$; that is, (1.3) holds for all $x, y \in \Omega$;

(ii) there exists $\delta>0$ such that for a.e. $x \in \Omega$ the matrix $D f(x)$ satisfies

$$
v^{T} D f(x) v \geq \delta|D f(x) v \| v| \quad \text { for every vector } v \in \mathbf{R}^{n}
$$

(iii) there exists $\gamma>0$ such that for a.e. $x \in \Omega$ the matrix $D f(x)$ satisfies

$$
v^{T} D f(x) v \geq \gamma\|D f(x)\||v|^{2} \quad \text { for every vector } v \in \mathbf{R}^{n} .
$$

The constants $\delta$ and $\gamma$ depend only on each other. 
Proof. The equivalence of (i) and (ii), with the same constant $\delta$, was proved in [12, p. 397]. It is obvious that (iii) implies (ii) with $\delta=\gamma$. It remains to establish the converse implication (ii) $\Longrightarrow$ (iii). To this end we need the following

Claim: If a real square matrix $A$ satisfies

$$
v^{T} A v \geq \delta|A v \| v| \quad \text { for every } v \in \mathbf{R}^{n},
$$

then

$$
|A v| \geq c\|A\||v| \quad c=c(\delta)>0 .
$$

Although this claim is known, even with a sharp constant [3], we give a proof for the sake of completeness. It suffices to estimate $|A v|$ from below under the assumptions that $A v \neq 0$ and $\|A\|=1=|v|$. Let $u$ be a unit vector in $\mathbf{R}^{n}$ such that $|A u|=1$. Replacing $u$ by $-u$ if necessary we may assume that $u^{T} A v+v^{T} A u \leq 0$. Let $\lambda=$ $\sqrt{|A v|}$. On one hand we have

$$
(\lambda u+v)^{T} A(\lambda u+v) \leq \lambda^{2} u^{T} A u+v^{T} A v \leq \lambda^{2}+\lambda^{2}=2 \lambda^{2} .
$$

On the other hand

$$
(\lambda u+v)^{T} A(\lambda u+v) \geq \delta|\lambda A u+A v||\lambda u+v| \geq \delta\left(\lambda-\lambda^{2}\right)(1-\lambda) .
$$

Combining (2.2) and (2.3) we obtain $2 \lambda \geq \delta(1-\lambda)^{2}$, hence

$$
\lambda \geq \delta^{-1}+1-\sqrt{\left(\delta^{-1}+1\right)^{2}-1}>0 .
$$

This proves the claim.

\section{Delta-monotone mappings and doubling measures}

The following result shows that $\mathscr{D} \mathscr{M} \subset \mathscr{Q} \mathscr{C}$. In particular, $f \in \mathscr{D} \mathscr{M}$ implies that $f$ is a continuous Sobolev mapping, and therefore (ii)-(iii) of Lemma 2.2 hold.

Proposition 3.1. [12, Theorem 6] Every nonconstant $\delta$-monotone mapping is $\eta$-quasisymmetric where $\eta$ depends only on $\delta$.

It is well-known that quasisymmetric mappings are closely related to doubling measures [11]. The following lemma is another instance of this relation.

Lemma 3.2. For any nonconstant $\delta$-monotone mapping $f: \mathbf{R}^{n} \rightarrow \mathbf{R}^{n}(n \geq 2)$ the measure $\mu=\|D f(x)\| d x$ is doubling. The doubling constant $\mathscr{D}_{\mu}$ depends only on $\delta$ and $n$.

Proof. Recall that $f$ is quasisymmetric. Lemma 3.2 in [14] implies the existence of a constant $C=C(\delta, n)$ such that

$$
C^{-1} \frac{\operatorname{diam} f(B)}{\operatorname{diam} B} \leq \frac{1}{|B|} \int_{B}\|D f\| d x \leq C \frac{\operatorname{diam} f(B)}{\operatorname{diam} B}
$$

for all balls $B \subset \mathbf{R}^{n}$. Since $\operatorname{diam} f(2 B) \leq C \operatorname{diam} f(B)$ with $C=C(\eta)$, the lemma follows.

Recall that $\phi: \mathbf{R}^{n} \rightarrow(0, \infty)$ is the Gaussian kernel (1.4). Let $\mathbf{B}=B(0,1)$ be the open unit ball in $\mathbf{R}^{n}$. 
Lemma 3.3. Let $\mu$ be a doubling measure in $\mathbf{R}^{n}$ and $p \geq 0$. Let $\Omega$ be either $\mathbf{R}^{n}$ or the half space $\{y:\langle y, \xi\rangle \geq 0\}$ for some $\xi \in \mathbf{R}^{n}$. Then

$$
C^{-1} \mu(\mathbf{B}) \leq \int_{\Omega}|y|^{p} \phi(y) d \mu(y) \leq C \mu(\mathbf{B})
$$

where the constant $C$ depends only on $\mathscr{D}_{\mu}, p$ and $n$.

Proof. We begin by estimating the integral in (3.2) from above as follows

$$
\int_{\mathbf{R}^{n}}|y|^{p} \phi(y) d \mu(y)=\int_{\mathbf{B}}|y|^{p} \phi(y) d \mu(y)+\sum_{k=0}^{\infty} \int_{2^{k}<|y| \leq 2^{k+1}}|y|^{p} \phi(y) d \mu(y),
$$

where

$$
\int_{\mathbf{B}}|y|^{p} \phi(y) d \mu(y) \leq \phi(0) \mu(\mathbf{B})=(2 \pi)^{-\frac{n}{2}} \mu(\mathbf{B})
$$

and

$$
\begin{aligned}
\int_{2^{k}<|y| \leq 2^{k+1}}|y|^{p} \phi(y) d \mu(y) & \leq 2^{p(k+1)}(2 \pi)^{-\frac{n}{2}} e^{-2^{2 k-1}} \mu\left(B\left(0,2^{k+1}\right)\right) \\
& \leq 2^{p(k+1)}(2 \pi)^{-\frac{n}{2}} e^{-2^{2 k-1}} \mathscr{D}_{\mu}^{k+1} \mu(\mathbf{B}) .
\end{aligned}
$$

Summing over $k=0,1,2 \ldots$ we obtain

$$
\int_{\mathbf{R}^{n}} \phi(y) d \mu(y) \leq C \mu(\mathbf{B})
$$

where $C=C\left(\mathscr{D}_{\mu}, p, n\right)>0$.

We turn to the left side of (3.2). The inequality

$$
|y|^{p} \phi(y) \geq \frac{e^{-1 / 2}}{2^{p}(2 \pi)^{n / 2}} \quad \text { for } \frac{1}{2} \leq|y| \leq 1
$$

implies

$$
\int_{\Omega}|y|^{p} \phi(y) d \mu(y) \geq \frac{e^{-1 / 2}}{2^{p}(2 \pi)^{n / 2}} \mu(\Omega \cap\{1 / 2 \leq|y| \leq 1\}) .
$$

Since $\mu(\Omega \cap\{1 / 2 \leq|y| \leq 1\}) \geq \mathscr{D}_{\mu}^{-3} \mu(\mathbf{B})$, the left side of (3.2) follows.

\section{Proof of main results}

Proof of Theorem 1.3. Since $f$ is quasisymmetric by Proposition 3.1, it satisfies the growth condition $|f(x)| \leq \alpha|x|^{p}+\beta$ for some constants $\alpha, \beta, p$, see [11, Theorem 11.3]. Therefore, the integrals (1.5) and (1.6) converge and $F$ is $C^{\infty}$-smooth in $\mathbf{H}^{n+1}$. Let $\gamma=\gamma(\delta)>0$ be as in part (iii) of Lemma 2.2 .

Our first step is to prove that for $(x, t) \in \mathbf{H}^{n+1}$ the matrix $\mathscr{B}:=D F(x, t)$ satisfies the condition

$$
w^{T} \mathscr{B} w \geq \gamma_{1}\|\mathscr{B}\||w|^{2} \quad \text { for every vector } w \in \mathbf{R}^{n+1}
$$


where $\gamma_{1}=\gamma_{1}(\delta, n)>0$. Fix $x \in \mathbf{R}^{n}$ and $t>0$. We compute the partial derivatives of $F$ at $(x, t) \in \mathbf{H}^{n+1}$ as follows:

$$
\begin{aligned}
\frac{\partial F^{i}}{\partial x_{j}} & =\int_{\mathbf{R}^{n}} f_{j}^{i}(x+t y) \phi(y) d y, \quad 1 \leq i, j \leq n ; \\
\frac{\partial F^{i}}{\partial t} & =\int_{\mathbf{R}^{n}} \sum_{j=1}^{n} f_{j}^{i}(x+t y) y^{i} \phi(y) d y, \quad 1 \leq i \leq n ; \\
\frac{\partial F^{n+1}}{\partial x_{j}} & =\int_{\mathbf{R}^{n}} \sum_{i=1}^{n} f_{j}^{i}(x+t y) y^{j} \phi(y) d y, \quad 1 \leq j \leq n ; \\
\frac{\partial F^{n+1}}{\partial t} & =\int_{\mathbf{R}^{n}} \sum_{i=1}^{n} \sum_{j=1}^{n} f_{j}^{i}(x+t y) y^{i} y^{j} \phi(y) d y .
\end{aligned}
$$

To simplify formulas we write $A(y)=D f(x+t y)$ and let $B(y)$ be the $(n+1) \times(n+1)$ matrix written in block form below,

$$
B(y)=\left(\begin{array}{c:c}
A(y) & A(y) y \\
\hdashline y^{T} A(y) & y^{T} A(y) y
\end{array}\right) .
$$

With this notation we have

$$
D F(x, t)=\int_{\mathbf{R}^{n}} B(y) \phi(y) d y .
$$

First we show that the norm of $\mathscr{B}$ is dominated by the quantity

$$
\alpha:=\int_{B(0,1)}\|A(y)\| d y
$$

Indeed,

$$
\|\mathscr{B}\| \leq \int_{\mathbf{R}^{n}}\|B(y)\| \phi(y) d y \leq \int_{\mathbf{R}^{n}}\|A(y)\|(1+|y|)^{2} \phi(y) d y .
$$

By Lemma 3.2 the measure $\mu=\|A(y)\| d y$ is doubling. Applying Lemma 3.3 we obtain

$$
\|\mathscr{B}\| \leq C \alpha, \quad C=C(\delta, n) .
$$

Next we estimate the quadratic form $w \mapsto w^{T} \mathscr{B} w$ generated by $\mathscr{B}$ from below. For this we fix a vector $w \in \mathbf{R}^{n+1}$, written as $w=v+s e_{n+1}$ with $v \in \mathbf{R}^{n}$ and $s \in \mathbf{R}$. It is easy to see that

$$
w^{T} B(y) w=(v+s y)^{T} A(y)(v+s y) .
$$

Let $\Omega=\left\{y \in \mathbf{R}^{n}:\langle v, s y\rangle \geq 0\right\}$. Then

$$
\begin{aligned}
w^{T} \mathscr{B} w & =\int_{\mathbf{R}^{n}}\left\{(v+s y)^{T} A(y)(v+s y)\right\} \phi(y) d y \\
& \geq \gamma \int_{\mathbf{R}^{n}}\|A(y)\||v+s y|^{2} \phi(y) d y \\
& \geq \gamma \int_{\Omega}\|A(y)\||v+s y|^{2} \phi(y) d y \\
& \geq \gamma|v|^{2} \int_{\Omega}\|A(y)\| \phi(y) d y+\gamma s^{2} \int_{\Omega}\|A(y)\||y|^{2} \phi(y) d y .
\end{aligned}
$$


Applying Lemma 3.3 with $\mu=\|A(y)\| d y$ we obtain

$$
w^{T} \mathscr{B} w \geq c \alpha \gamma\left(|v|^{2}+s^{2}\right)=c \alpha \gamma|w|^{2}, \quad c=c(\delta, n) .
$$

Combining (4.4) and (4.5) we obtain (4.1) with $\gamma_{1}=(c / C) \gamma$. By virtue of Lemma 2.2 $F$ is $\delta_{1}$-monotone in the upper half-space $\mathbf{H}^{n+1}$ where $\delta_{1}=\delta_{1}(\delta, n)$. By symmetry, $F$ is also $\delta_{1}$-monotone in the lower half-space.

To prove that $F$ is $\delta_{1}$-monotone in the entire space $\mathbf{R}^{n+1}$, we consider two points $a, b \in \mathbf{R}^{n+1}$ such that the line segment $[a, b]$ crosses the hyperplane $\mathbf{R}^{n}$ at some point c. We have

$$
\begin{aligned}
\langle F(a)-F(b), a-b\rangle & =\langle f(a)-f(c), a-b\rangle+\langle F(c)-F(b), a-b\rangle \\
& \geq \delta_{1}|F(a)-F(c)||a-b|+\delta_{1}|F(c)-F(b)||a-b| \\
& \geq \delta_{1}|F(a)-F(b)||a-b| .
\end{aligned}
$$

Therefore, $F \in \mathscr{D} \mathscr{M}$.

It remains to show that $F: \mathbf{H}^{n+1} \rightarrow \mathbf{H}^{n+1}$ is bi-Lipschitz in the hyperbolic metric. Since $F \in \mathscr{Q} \mathscr{C}$ and $\mathbf{H}^{n+1}$ is a geodesic space, it suffices to prove that

$$
\|D F(x, t)\| \approx \frac{F^{n+1}(x, t)}{t} .
$$

Here $X \approx Y$ means that $X$ and $Y$ are comparable, i.e., $C^{-1} Y \leq X \leq C Y$ where $C=C(\delta, n)$. It follows from (4.4) and (4.5) that $\|D F(x, t)\|$ is comparable to the integral average of $\|D f\|$ over the ball $B(x, t)$. By (3.1) this average is comparable to $t^{-1}$ diam $f(B(x, t))$. The quasisymmetry of $F$ implies (cf. [11, 11.18])

$$
\operatorname{diam} f(B(x, t)) \approx|F(x, t)-F(x, t / 2)| \approx F^{n+1}(x, t) .
$$

This proves (4.6).

Proof of Proposition 1.4. The proof of Theorem 1.3 also works in the case $n=1$ with the following interpretation. Since quasisymmetric mappings on the line need not be absolutely continuous [4], the derivative $f^{\prime}$ must be understood in the sense of distributions. In fact, $\mu:=f^{\prime}$ is a positive doubling measure with $\mathscr{D}_{\mu}=\mathscr{D}_{\mu}(\eta)[11$, 13.20]. Lemma 3.2 is not needed in this case. The rest of the proof carries over with $\gamma=1$ and $\gamma_{1}=\gamma_{1}\left(\mathscr{D}_{\mu}\right)$.

Proof of Corollary 1.5. According to [12, Lemma 18], a $K$-quasiconformal mapping with a convex potential is also $\delta$-monotone with $\delta=\delta(K, n)$. Let $F$ be the $\delta_{1}$-monotone extension of $f$ provided by Theorem 1.3. Since the differential matrix $D f$ is symmetric, the formulas (4.2) and (4.3) show that $D F$ is symmetric as well. In addition, $D F$ is positive semidefinite by Lemma 2.2. Thus, $F=\nabla U$ for some convex function $U: \mathbf{R}^{n+1} \rightarrow \mathbf{R}$.

\section{Concluding remarks}

Corollary 1.2 naturally leads one to wonder how large the group $\mathscr{Q} \mathscr{C}_{d}$ really is. It is easy to give an example of a planar quasiconformal mapping that cannot be factored into delta-monotone mappings. Indeed, the logarithmic spiral map

$$
S\left(\rho e^{i \theta}\right)=\rho e^{i(\theta+\log \rho)}
$$

has this property, which can be verified as follows. Let us say that a simple curve $\Gamma:[0,1] \rightarrow \mathbf{C}$ is of bounded twist if for any $a \in[0,1]$ there is a bounded continuous 
branch of the function $t \mapsto \arg (\Gamma(t)-\Gamma(a)), t \in[0,1] \backslash\{a\}$. For any delta-monotone mapping $F: \mathbf{C} \rightarrow \mathbf{C}$ the principal value of

$$
\arg \frac{F(z)-F(w)}{z-w}, \quad z, w \in \mathbf{C}, z \neq w
$$

defines a bounded continuous function on $\mathbf{C}^{2} \backslash\{z=w\}$. It follows that the image of a curve of bounded twist is invariant under delta-monotone mappings. But the image of the line segment $[0,1]$ under the mapping $S$ in $(5.1)$ is not of bounded twist.

It is not as easy to give an example of an element in $\mathscr{Q} \mathscr{C} \backslash \mathscr{Q} \mathscr{C}{ }_{d}$. For instance, the mapping $S$ belongs to $\mathscr{Q} \mathscr{C}_{d}$ by virtue of being bi-Lipschitz. This motivates the following question:

Question 5.1. What are the obstructions for factorization of a quasiconformal mapping into bi-Lipschitz and delta-monotone factors?

Inspired by the example (5.1), one could expect to find such an obstruction in the form of a $\mathscr{Q} \mathscr{C}_{d}$-invariant property of curves. For instance, both bi-Lipschitz and delta-monotone mappings take smooth curves into rectifiable curves [2, Theorem 3.11.7]. However, this is no longer true of their compositions; in fact, the image of a line segment under a planar $\mathscr{Q}_{d}$ mapping can have Hausdorff dimension arbitrarily close to 2 . We briefly sketch this construction,leaving details to the reader. The first step is to find a bi-Lipschitz mapping $g: \mathbf{R}^{2} \rightarrow \mathbf{R}^{2}$ such that $g(\mathbf{R})$ contains a planar Cantor set $E$ of dimension $0<\beta<1$ (see Lemma 3.1 [5] and the comment after its proof). Then one constructs a delta-monotone mapping $h: \mathbf{R}^{2} \rightarrow \mathbf{R}^{2}$ such that the Hausdorff dimension of $h(E)$ is equal to $\alpha$, for a given $\alpha \in(0,2)$ (see the proof of [10, Theorem 5]). Finally, let $f=h \circ g$.

Acknowledgements. We thank Mario Bonk and Jang-Mei Wu for conversations related to the subject of this paper.

\section{References}

[1] Ahlfors, L. V.: Extension of quasiconformal mappings from two to three dimensions. - Proc. Natl. Acad. Sci. USA 51, 1964, 768-771.

[2] Astala, K., T. Iwaniec, and G. J. Martin: Elliptic partial differential equations and quasiconformal mappings in the plane. - Princeton Univ. Press, Princeton, 2009.

[3] Astala, K., T. Iwaniec, and G. J. Martin: Monotone maps of $\mathbf{R}^{n}$ are quasiconformal. Methods Appl. Anal. 15:1, 2008, 31-38.

[4] Beurling, A., and L. Ahlfors: The boundary correspondence under quasiconformal mappings. - Acta Math. 96, 1956, 125-142.

[5] Bishop, C. J.: Quasiconformal mappings which increase dimension. - Ann. Acad. Sci. Fenn. Math. 24:2, 1999, 397-407.

[6] Brenier, Y.: Polar factorization and monotone rearrangement of vector-valued functions. Comm. Pure Appl. Math. 44, 1991, 375-417.

[7] Caffarelli, L. A.: The regularity of mappings with a convex potential. - J. Amer. Math. Soc. 5:1, 1992, 99-104.

[8] Carleson, L.: The extension problem for quasiconformal mappings. - In: Contributions to analysis, edited by L. V. Ahlfors et al., Academic Press, New York, 1974, 39-47.

[9] Fefferman, R. A., C. E. Kenig, and J. Pipher: The theory of weights and the Dirichlet problem for elliptic equations. - Ann. of Math. (2) 134, 1991, 65-124. 
[10] Gehring, F. W., and J. VÄIsÄLÄ: Hausdorff dimension and quasiconformal mappings. - J. London Math. Soc. (2) 6, 1973, 504-512.

[11] Heinonen, J.: Lectures on analysis on metric spaces. - Universitext, Springer-Verlag, New York, 2001.

[12] Kovalev, L. V.: Quasiconformal geometry of monotone mappings. - J. Lond. Math. Soc. (2) $75: 2,2007,391-408$.

[13] Kovalev, L. V., and D. Maldonado: Mappings with convex potentials and the quasiconformal Jacobian problem. - Illinois J. Math. 49:4, 2005, 1039-1060.

[14] Kovalev, L. V., D. Maldonado, and J.-M. Wu: Doubling measures, monotonicity, and quasiconformality. - Math. Z. 257:3, 2007, 525-545.

[15] Sullivan, D.: Hyperbolic geometry and homeomorphisms. - In: Geometric topology, edited by J. C. Cantrell, Academic Press, New York-London, 1979, 543-555.

[16] Tukia, P., and J. VäısÄLÄ: Quasiconformal extension from dimension $n$ to $n+1$. - Ann. of Math. (2) 115:2, 1982, 331-348.

[17] VÄISÄLÄ, J.: Lectures on $n$-dimensional quasiconformal mappings. - Lecture Notes in Math. 229, Springer-Verlag, Berlin-New York, 1971.

Received 4 June 2010 